\title{
LA BIBLIA SOBRE EL TEJADO. \\ EN TORNO A LOS TEXTOS BÍBLICOS INSCRITOS \\ EN TEGULAE Y LATERES*
}

\author{
Javier Velaza \\ Universidad de Barcelona \\ javier.velaza@gmail.com
}

\section{RESUMEN}

Este trabajo revisa las inscripciones realizadas sobre tegulae o lateres con textos bíblicos y analiza su función. Contra lo que a menudo se ha pensado, no se trata de inscripciones funerarias, sino apotropaicas o de consagración.

PALABRAS ClAVE: Epigrafía, instrumentum, tegulae, lateres, Biblia, religión.

THE BIBLE ON THE ROOF.

AROUND THE BIBLICAL TEXTS INSCRIBED ON TEGULAE AND LATERES

\section{ABSTRACT}

This paper reviews the inscriptions made on tegulae or lateres with biblical texts and analyzes their function. Contrary to what has often been thought, these are not funeral inscriptions, but rather apotropaic or consecratory.

KEYWORDS: Epigraphy, instrumentum, tegulae, lateres, Bible, religion.

En el contexto de la amplísima presencia epigráfica de la Biblia, estudiada de manera ejemplar por Antonio Enrico Felle ${ }^{1}$, los ejemplares sobre tegulae o lateres constituyen un subgrupo no muy abundante pero de notable interés. Más allá de su eventual valor como testimonio de transmisión indirecta del texto de las Escrituras -cuestión en la que no entraremos en estas páginas-, el principal problema que este tipo de inscripciones presenta es el de comprender su función, que generalmente se ha supuesto sepulcral. Nuestro propósito en este trabajo es replantear tal hipótesis, para lo que previamente será necesario llevar a cabo una revisión y actualización de las inscripciones conocidas hasta el momento.

Felle recogió en su Biblia epigraphica solamente seis ejemplares, a los que han de añadirse ahora dos más publicados con posterioridad a su trabajo. Así, el repertorio está constituido por los siguientes epígrafes: 


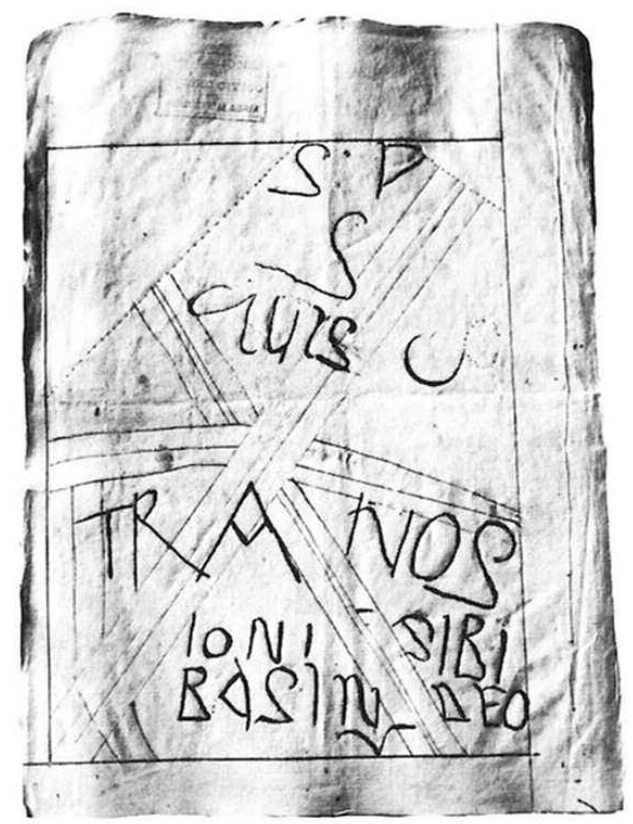

Fig. 1: Teja de Lazzàro (calco de Morisano (según ICI v 3)).

1. Lazzàro (fig. 1).

Bibl.: BE 641; CIL x 15; ICI v 3; ILCV 2489, +2195, +2485.

Teja mutilada por su parte superior izquierda y derecha. Inscripción realizada antes de la cocción de la pieza, a lo que parece sobre una decoración en forma de cruz o de chrismon. Datable, según Felle, a finales del s. VI o inicios del s. VII.

[Si deu]s p [ro]

[nobi]s

quis $\operatorname{co}(\mathrm{n})$ -

tra nos

[D]ionisi bi-

bas in deo

* Este trabajo ha sido realizado en el marco del proyecto PID2019-105650GB-I00 y del Grup de Recerca Consolidat LITTERA (2017SGR241).

${ }^{1}$ Biblia epigraphica. La sacra scrittura nella documentazione epigrafica dell Orbis Christianus Antiquus (III-VIII seccolo), Bari 2006 (en adelante BE). 


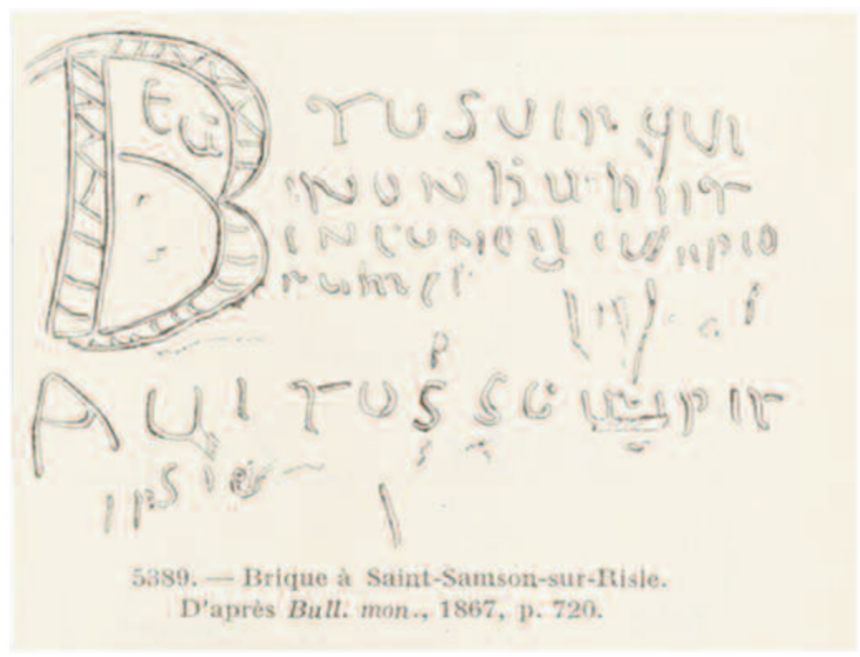

Fig. 2: Teja de Saint-Samson-de-la-Roque (según De Caumont).

El epígrafe recoge en su primera parte $\mathrm{Rm} 8,31$ (Quid ergo discemus adversus haec? Si Deus pro nobis, quis contra nos?). Felle interpreta el epígrafe como sepulcral, aunque, como se dirá después, no sin algunas dudas. También Buonocore (ICI v 3) lo supone "iscrizione funeraria su mattone destinato a tomba".

2. Saint-Samson-de-la-Roque, monasterio de Pental (fig. 2).

Bibl.: BE 769; De Caumont 1858, pp. 39-40; De Caumont 1867, pp. 719-720; Leclercq 1925, col. 1538, fig. 5389; Mallon 1948, p. 123; Mallon 1952a, p. 54 n. 65; Charlier 2004, 27.2.

Ladrillo. Inscripción realizada antes de cocción. Datable en el s. VI.

Beatus vir qui

non habiit

in concilio impio-

rum

Avitus sc $<$ ri $>$ psit

5

ipse

El ipse de la l. 6 no fue editado por Felle, pero fue correctamente añadido por Charlier.

El epígrafe reproduce en su primera parte el inicio de Sal 1,1: beatus vir qui non abiit in consilio impiorum, et in via peccatorum non stetit, et in cathedra pestilentiae non sedit.

Felle interpreta el epígrafe como "forse sepolcrale". 


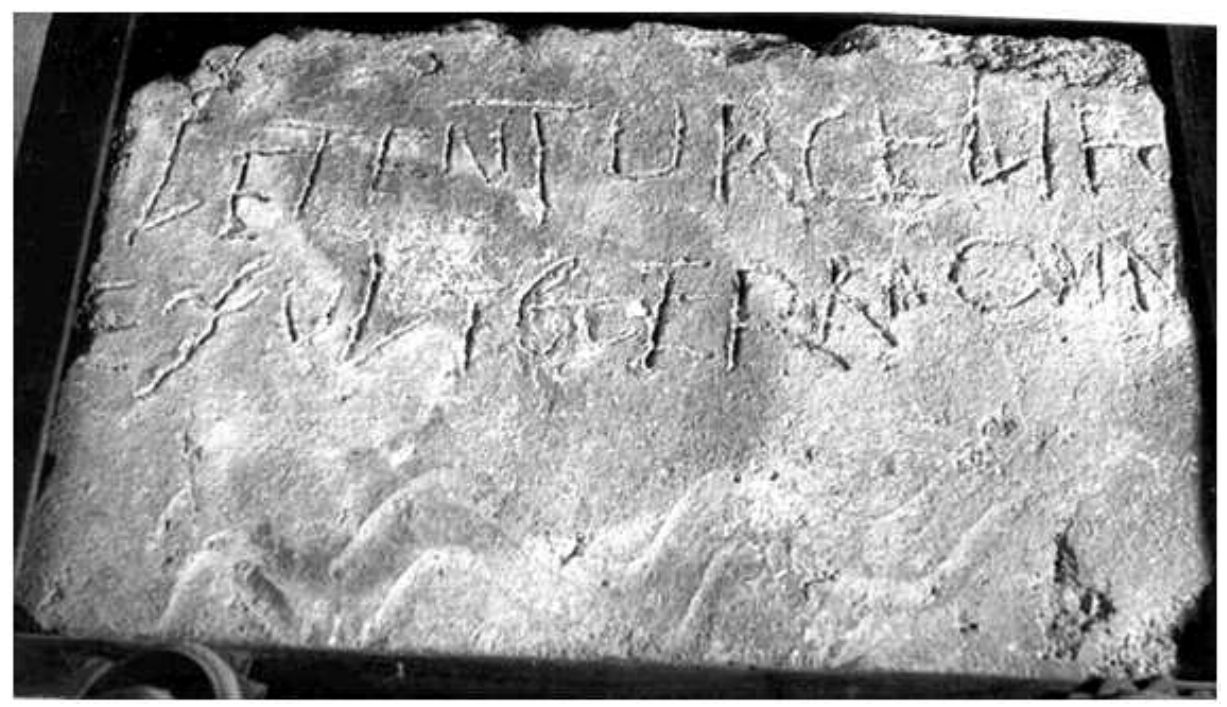

Fig. 3: Teja de Villaviciosa de Córdoba (foto http://www3.uah.es/imagines cilii/fotos cilii/7/CILII7,0700.jpg).

3. Villaviciosa de Córdoba (fig. 3).

Bibl.: BE 780; CIL II²/7, 700; ICERV 415; AE 1953, 265; ILER 415; Romero de Torres 1919, pp. 375-377; Mallon 1948, p. 123; Mallon 1952a, pp. 207-216; CLHisp 181g; Escolà - Martínez Gázquez 2002, p. 237 n. 24.

Teja. Inscripción realizada antes de cocción. Final del s. IV o comienzo del s. V.

Letentur celi et exultet terra omn(is)

Las últimas letras de 1. 2 han sido interpretadas por la mayor parte de los editores como (c)omm [oveatur, sin duda por el modelo de Sal 95,11 (Laetentur coeli et exsultet terra, commoveatur mare, et plenitudo eius), al que el epígrafe responde. Sin embargo, las letras inscritas en la teja son inequívocamente OMN, como ya vio su primer editor, Romero de Torres, cuya lectura e hipótesis de resolución creemos que hay que recuperar.

4. Barcelona (fig. 4).

Bibl.: Mallon 1948, p. 124; Mallon 1952a, p. 64 n. 66; BE 786; IRC IV, 309; IRC v, p. 116; CLEHisp 181f.

Teja. Inscripción realizada antes de cocción. S. VII.

[E psal]mis Davidis quis loquitur pote[nti-] 


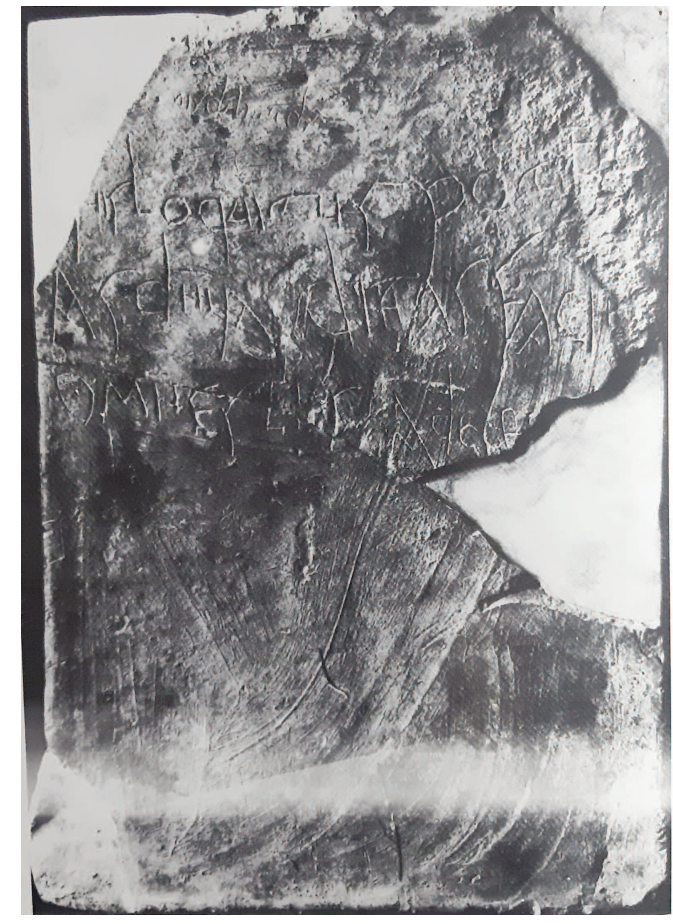

Fig. 4: Teja de Barcelona (foto IRC IV).

as $\mathrm{d}$ (omi)ni auditas facie[t] omnes eius $\mathrm{l} a<\mathrm{u}>$ des e[ius] NS

Como señala Felle, las letras NS de 1. 5 tal vez hayan de interpretarse como IVS, de modo que constituirían la secuencia final de la palabra de la línea anterior. El epígrafe reproduce Sal 105,2: quis loquetur potentias Domini: auditas faciet omnes laudes eius?

Para Mallon y para Felle, se trataría también de un epígrafe sepulcral, aunque con reparos en el caso de este último.

5. Saint Maurice d'Agaune (figg. 5 y 6 ).

Bibl.: BE 776 = CIMAH I, 23, tab. 13, figg. 30-31.

Teja. Inscripción realizada probablemente antes de cocción. Final del s. VI $\mathrm{o}$ inicios del s. VII.

Dominus reget $\mathrm{m}[\mathrm{e}$ e]t nihil mihi dierit in loco $\mathrm{pa}(\mathrm{s})$ $\mathrm{cu}[\mathrm{ae}] \mathrm{i}$ 


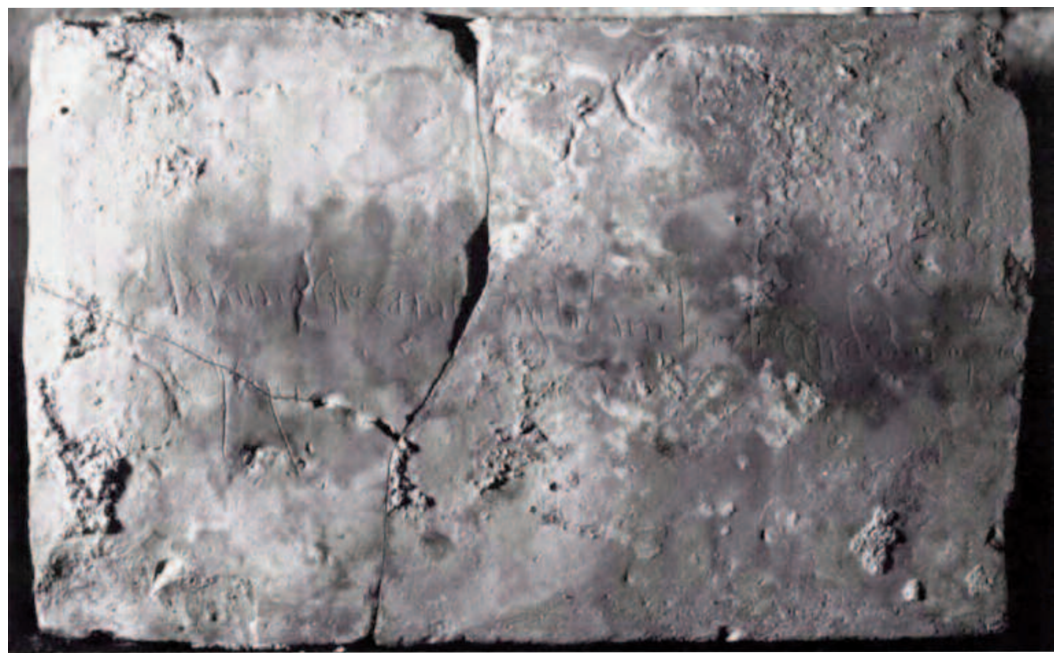

Fig. 5: Teja de Saint Maurice d'Agaune (foto CIMAH).

La inscripción se interrumpe en 1.2 a partir de la letra I. El texto corresponde a Sal 22, 1-2: Dominus reget me et nibil mibi deerit. In loco pascuae ibi me conlocavit. Super aquam refectionis educavit me.

Felle lo considera un epígrafe sepulcral.

\section{La Zubia (Granada) (fig. 7).}

Bibl.: BE 779; ILPGranada 162; IHC 375; CILA IV, 63.

Teja. Inscripción realizada antes de cocción. Finales del s. VIII o inicios del s. IX.

((Crux)) Pauperes vobiscum abebitis me autem senper vobiscum non avebitis qui legis intellige

Es posible identificar dos textos bíblicos: en 11. 1-3, Mt 26,11: Nam semper pauperes habebitis vobiscum, me autem non semper habebitis; y en 1l. 3-4 Mt 24,15: Cum ergo videritis abominationem desolationis, quae dicta est a Daniele propheta, stantem in loco sancto: qui legit intelligat.

Según Felle, se trataría también de un epígrafe funerario.

7. Vranje - Prvonek (Serbia) (fig. 8).

Bibl.: Nedeljkovi 2006, pp. 377-378. 380, n. 1, fig. 1; AE 2006, 1187.

Teja. Inscripción realizada antes de cocción. 


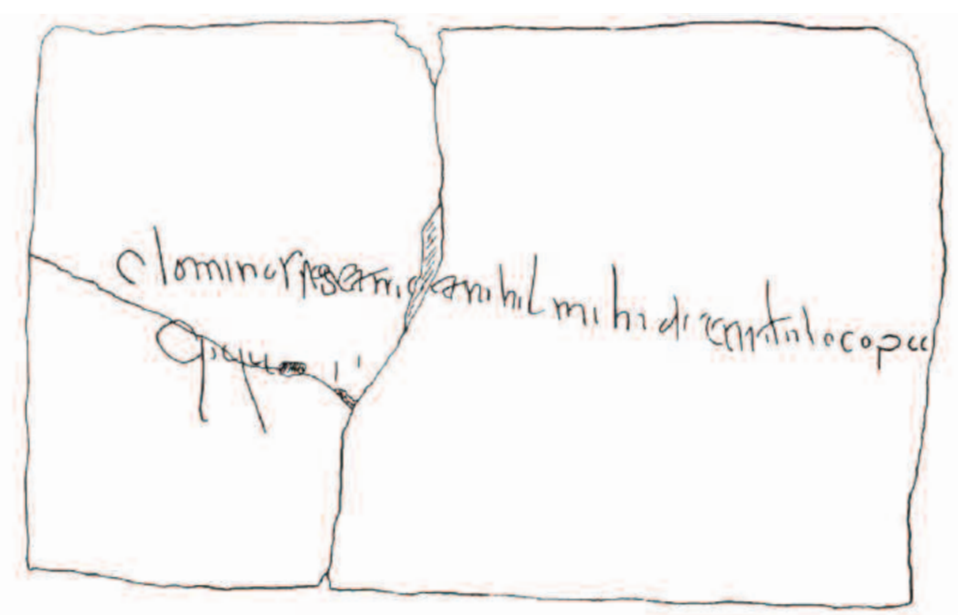

Fig. 6: Teja de Saint Maurice d'Agaune (dibujo CIMAH).

Exauda te Dominus

in die tribulationis

El texto corresponde a Sal. 19,2: Exaudiat te Dominus in die tribulationis: protegat te nomen Dei Iacob.

El editor señala que "These inscriptions provide new evidence of what seems to have been common practice among ancient Christian builders: messages written on tiles or bricks (...) used to travel from the brick plant over to the building site containing prayers and good wishes, to the attention of the masons, who were able to read them before putting the material into final use".

8. Portimão (fig. 9).

Bibl.: Marques - Lopes - Liberato 2016, 580; AE 2016, 661.

Teja (imbrex). Inscripción realizada antes de la cocción. Tal vez del s. IV o V.

[-]us dei gratia a[-]

[inclin]a aurem tuam et [exaudi me - - ]

$[-] \mathrm{re}++++[-$

En su parte mejor identificable, el texto corresponde con Sal 85,1: Inclina, Domine, aurem tuam et exaudi me, quoniam inops et pauper sum ego.

A partir de la actualización del corpus de inscripciones, conviene subrayar algunos de los rasgos comunes a todos los ejemplares conocidos. En primer lugar, destaca el hecho de que, prácticamente en todos los casos, se trata de inscripciones realizadas antes de la cocción de la pieza. Solamente en el caso del ejemplar n. 5 


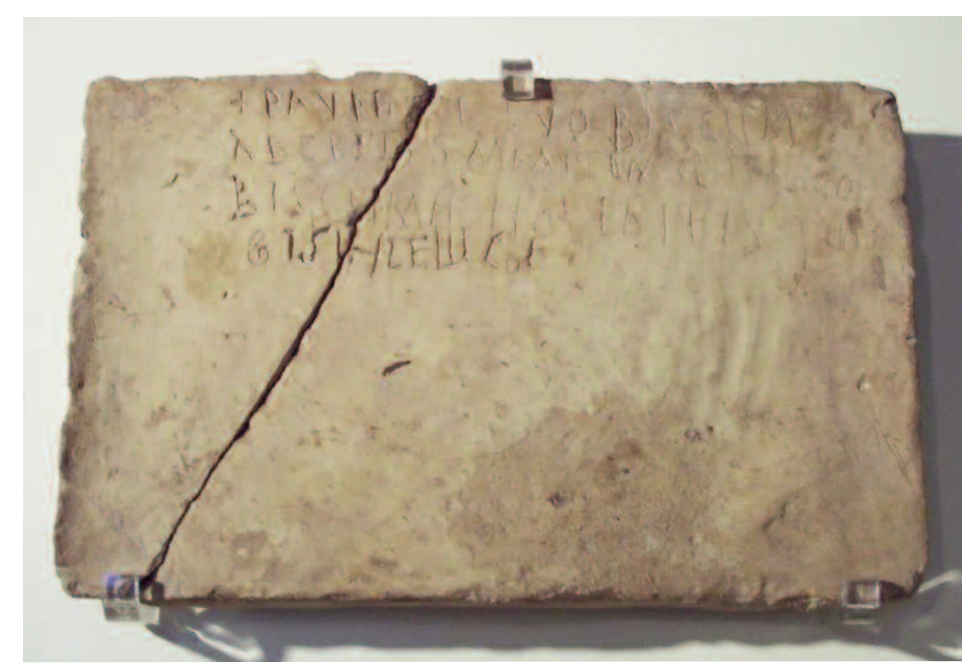

Fig. 7: Teja de La Zubia (Granada)

(foto Inmaculada de la Torre Castellano (http://ceres.mcu.es/)).

(Saint Maurice d'Agaune) caben ciertas dudas al respecto 2 , pero, a juzgar por la fotografía publicada, parece probable que sea también un epígrafe inciso en crudo. En tales circunstancias, resulta evidente que estamos delante de inscripciones practicadas en el interior de la figlina y que su intención estaba ya prevista en el momento de fabricar la teja o el ladrillo correspondiente. Ello las diferenciará, por lo tanto, de otros ejemplares sobre idénticos soportes cuya inscripción ha sido llevada a cabo con posterioridad a la cocción y que, por lo tanto, representan una utilización secundaria del material.

Por otra parte, es significativo señalar que, en buena parte de los casos, el texto se reduce a la cita -más o menos correcta y completa- del pasaje testamentario, sin otras menciones: hacen excepción solamente la teja de Lazzarò (n. 1), que incluye la frase Dyonisi bibas in deo, y la de Saint-Samson-de-la-Roque (n. 2), que menciona al autor del epígrafe en la forma Avitus scripsi ipse.

Esta última constatación nos conduce al problema fundamental del conjunto, a saber, el de cuál es la función y el propósito de tales inscripciones. Como hemos dicho, existe una tendencia general a considerarlas como inscripciones funerarias, pero ello choca, a nuestro modo de ver, con varios obstáculos muy notables: el primero

${ }^{2}$ No hemos podido realizar autopsia de la pieza, que sería la prueba decisiva para determinar si la inscripción es pre- o postcocción. 


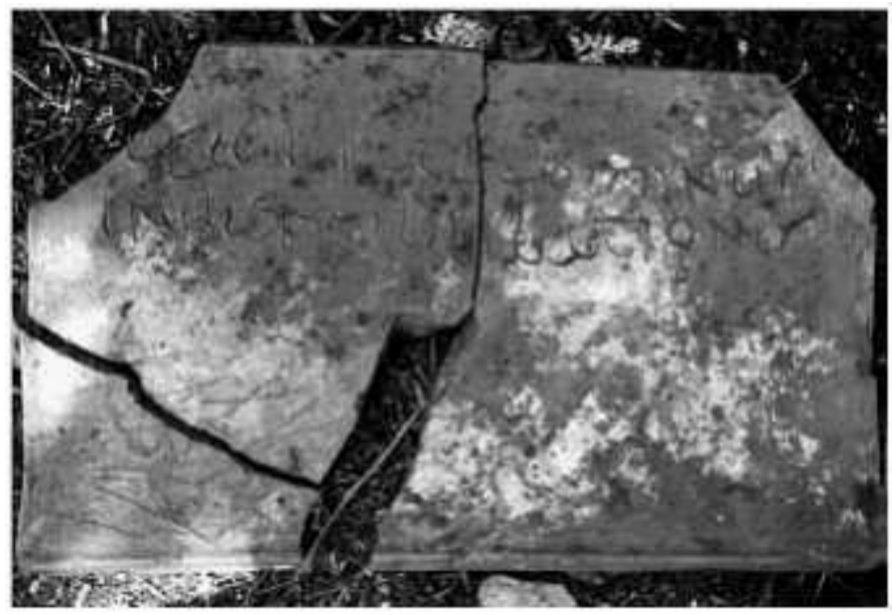

Fig. 8: Teja de Vranje - Prvonek (foto Nedeljković).

es, desde luego, el hecho de que en casi ninguno de los ejemplares aparezca un formulario sepulcral claro o, cuando menos, el nombre del difunto; el segundo vuelve a ser el que los textos han sido grabados en la figlina antes de la cocción de la pieza, lo que obligaría a considerar tales talleres como centros de producción de inscripciones sepulcrales, aspecto para el que carecemos de cualquier prueba o, siquiera, indicio; por otra parte, y aunque este sea solamente un argumento negativo y, en consecuencia, de menor peso, hay que observar que ninguna de las piezas conocidas procede con seguridad de una necrópolis o de un ámbito sepulcral.

Es cierto, en cualquier caso, que incluso aquellos autores que se decantan por considerarlas inscripciones funerarias expresan algunas dudas al respecto. Así, el propio Mallon, que en general les atribuía tal función, las calificaba en algún momento de “...monuments qui n'ont pas fait l'object de groupements matériels et dont l'intention et la destination se laissent mal deviner" ${ }^{3}$. Por su parte, Felle se muestra también favorable a la hipótesis sepulcral, pero demuestra reservas en casos como n. 3 y, en especial, en el n. 1, en el cual la mención de Rm 8, 31 le lleva a recordar que "il versetto, ricorrente nel corpus in altri ventuno casi, distribuiti piuttosto equamente tra Oriente e Occidente, trova diversi ambiti di uso, ma una sostanziale unità di funzione a scopo apotropaico (degli edifici di culto, delle fortificazioni, delle sepolture $)^{4}$.

${ }^{3}$ Mallon 1952b: 63-64.

${ }^{4}$ BE, p. 301. 


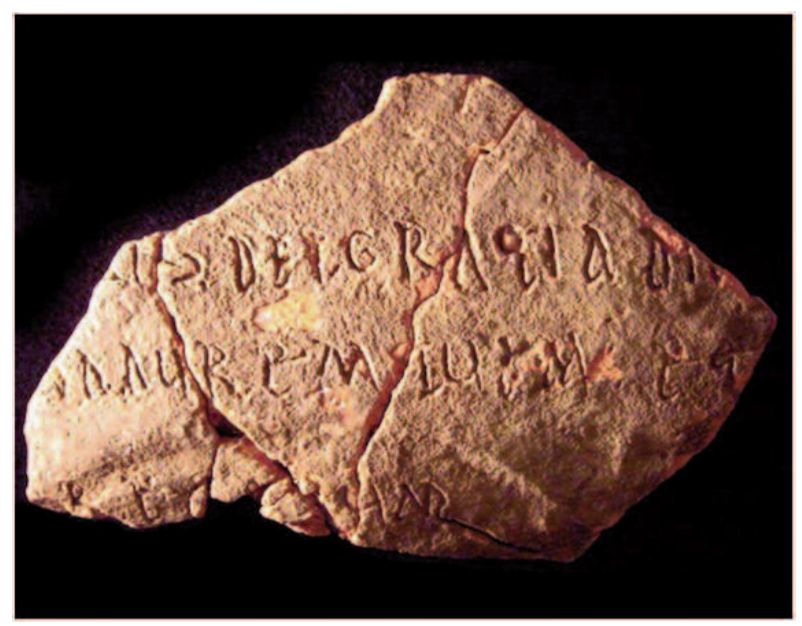

Fig. 9: Teja de Portimão (foto EDCS).

A nuestro modo de ver, para poder arrojar más luz sobre esta tipología inscriptoria, resulta imprescindible abrir el foco desde las piezas que documentan exclusivamente versículos bíblicos para insertarlas en un contexto más amplio y compararlas, cuando menos, con los ejemplares paralelos de época pagana realizados en soporte y con técnica similar. En este sentido, nos hemos ocupado no hace mucho de los epígrafes sobre teja y ladrillo con versos virgilianos ${ }^{5}$, que, a nuestro juicio, deben tener una función apotropaica o augural que ha de ponerse en relación con el bien conocido valor mágico que se atribuía al texto virgiliano ${ }^{6}$. Y también hemos dedicado algunas reflexiones a las tejas que presentan alfabetarios, otra tipología específicamente vinculada con ambientes religiosos o propiciatorios?.

En resumidas cuentas, todos estos tipos epigráficos parecen relacionados entre sí por una finalidad común, a saber, la de la consagración o la protección en términos religiosos de una obra o de un edificio. Se incluirían, desde este punto de vista, en la continuidad de una tradición que ha pervivido hasta tiempos recientes, porque todavía sobre tejas de los siglos XIX o XX aparecen plegarias o expresiones de bendición que los artesanos de las tejerías grabaron antes de la cocción de las piezas indudablemente con el objetivo de coronar la obra con buenos augurios.

${ }^{5}$ Velaza, 2016.

${ }^{6}$ Velaza, 2019a.

${ }^{7}$ Velaza, 2019b. 
En este orden de cosas, creemos que la aparición en dichos soportes de versículos o pasajes bíblicos - mayoritariamente pertenecientes a los Salmos, lo que resulta significativo, pero también al Nuevo Testamento-, debe de representar otro eslabón en esa misma tradición. De la misma manera que las sortes Biblicae sustituyeron a las sortes Vergilianae como texto adivinatorio, el texto de la Biblia vino a reemplazar al de la Eneida ${ }^{8}$ en su utilización como lenguaje apotropaico y protector de edificios sobre tejas y ladrillos?.

\section{REFERENCIAS BIBLIOGRÁFICAS}

$\mathrm{BE}=$ A. E. FelLE, Biblia Epigraphica. La sacra scrittura nella documentazione epigrafica dell Orbis Christianus Antiquus (III-VIII secolo), Bari 2006.

Castaño, J. M. (2018): "Las placas cerámicas decoradas tardoantiguas de la serie Bracario. Algunos apuntes y precisiones”, SPAL 27.1: 255-281.

Charlier, F. (2004): "La pratique de l'écriture dans les tuileries gallo-romaines", Gallia 6: 67-102.

$\mathrm{CIL}=$ Corpus Inscriptionum Latinarum, Berolini 1863-.

CILA IV = M. PASTOR, Corpus de Inscripciones Latinas de Andalucía. Granada, Sevilla 2002.

$\mathrm{CIMAH}=$ Corpus Inscriptionum Medii Aevi Helvetiae.

CLHisp = P. CugusI, Carmina Latina Epigraphica Hispanica post Buechelerianam collectionem editam reperta cognita (CLEHisp), Faenza 2012.

De Caumont, A. (1858): "Rapport verbal sur une excursión archéologique en Poitou et sur d'autres inspections faites dans le cours de l'année 1857”, Bulletin Monumental 24: 5-56.

De Caumont, A. (1867): “Nouvelles de l'Exposition”, Bulletin Monumental 33: 718-722.

EDCS = Epigraphische Datenbank Clauss-Slaby.

EscolÀ, J. M. - Martínez GázQueZ, J. (2002): "Tradición bíblica en los Carmina Latina Epigraphica”, en J. DE HoYo - J. GÓMeZ PALlarès (eds.), Asta ac pellege. 50 años de la publicación de Inscripciones Hispanas en Verso, de S. Mariner, Madrid, pp. 227-241.

ICERV = J. VIVES, Inscripciones cristianas de la España romana y visigoda, Barcelona 1969.

ICI = Inscriptiones Christianae Italiae septimo saeculo antiquiores.

ILCV = E. DIEHL, Inscriptiones Latinae Christianae Veteres, Berlin 1925-1967.

ILER = J. VIVES, Inscripciones latinas de la España romana, Barcelona, 1971-1972.

${ }^{8}$ Después de un periodo de convivencia entre ambos que seguramente hay que situar entre los siglos IV y V.

${ }^{9}$ No descartamos que en esta misma tradición puedan inscribirse tal vez los ladrillos visigóticos con decoración en relieve de crismones e invocaciones de los que conocemos numerosos ejemplares y cuya función es objeto de amplio debate (vid. últimamente Ruiz - Román, 2015 y Castaño, 2018). 
ILPGranada = M. PASTOR Muñoz - A. Mendoza EguARas, Inscripciones Latinas de la Provincia de Granada, Granada 1987.

IRC IV = G. FABRE - M. MAYER - I. RODÀ, Inscriptions romaines de Catalogne. IV. Barcino, Paris 1997.

LeClerCQ, H. (1925): “Graffites”, en DACL VI,2, París, coll. 1453-1542.

Mallon, J. (1948): "Filumene Asiana (Diehl, ILCV 2794)”, AEspA 70: 110-143.

MAllon, J. (1952a): "La brique de Villaviciosa de Córdoba (Ps. XCV, 11)", en Studi in onore di C. Manaresi, Milán, pp. 207-216.

Mallon, J. (1952b): Paléographie romaine, Madrid.

Romero de Torres, E. (1919): "Nuevo ladrillo visigótico con inscripción”, BRAE 74: 375-377.

NedelJKOVIĆ, V. (2006): "Rimski Grafitisa Gradišta kod Prvoneka (New roman graffiti from Gradište near Prvonek (Southern Serbia)”, Starinar 56: 377-380.

Marques, J. - Lopes, G. - Liberato, M. (2016): “Uma epígrafe paleocristã de Portimão”, Ficheiro epigráfico 141, n. 580.

RUIZ, J. I. - ROMÁN, J. M. (2015): “Las placas cerámicas decoradas tardoantiguas con iconografía cristiana en el sur de la península ibérica”, Anuario de Historia de la Iglesia Andaluza 3: 11-53.

VELAZA, J. (2016): "Virgilio en la figlina. Algunas reflexiones en torno a las inscripciones sobre teja y ladrillo con versos virgilianos", en J. CARBOnEll - H. Gimeno (eds.), A Baete ad fluvium Anam: Cultura epigráfica en la Bética Occidental y territorios fronterizos. Homenaje al profesor José Luis Moralejo Álvarez, Alcalá de Henares 2016, pp. 405-415.

VelazA, J. (2019a): "Las sortes Vergilianae, entre la realidad y la ficción”, en G. BARATTA - A. BUONOPANE - J. VELAZA (eds.), Cultura epigráfica y cultura literaria. Estudios en homenaje a Marc Mayer i Olivé, Bolonia, pp. 397-410.

VelAZA, J. (2019b): “Non solo lettere: l'alfabeto come elemento rituale nel mondo antico”, en G. BARATTA (ed.), L’ABC di un impero. Iniziare a scrivere a Roma, Roma, pp. 121-138. 